\title{
Chiral structure of the solutions of the Ginsparg-Wilson relation
}

\author{
Ting-Wai Chiu ${ }^{1}$, Chih-Wei Wang, Sergei V. Zenkin ${ }^{2}$ \\ Department of Physics, National Taiwan University, Taipei, 106 Taiwan, ROC \\ Received 7 July 1998 \\ Editor: M. Cvetič
}

\begin{abstract}
We analyse the structure of solutions of the Ginsparg-Wilson relation for lattice Dirac operator in topologically trivial gauge sector. We show that the properties of such solutions relating to the perturbative stability of the pole of the fermion propagator as well as to the structure of the Yukawa models based on these solutions are solely determined by the non-local chirally invariant part of these Dirac operators. Depending on the structure of this part, the pole in the fermion propagator may or may not be stable under radiative corrections. We illustrate this by explicit examples. (C) 1998 Elsevier Science B.V. All rights reserved.
\end{abstract}

\section{Introduction}

The Ginsparg-Wilson (GW) relation [1] for lattice Dirac operator opens very interesting new possibilities for formulation of vector gauge theories, in particular QCD, on a lattice [2-6]. In its simplest form the $\mathrm{GW}$ relation reads as

$D \gamma_{5}+\gamma_{5} D=2 r D \gamma_{5} D$,

where $D$ is the lattice Dirac operator and $r$ is a nonzero real parameter (the lattice spacing $a$ is set to one).

It is commonly regarded that Eq. (1) by itself ensures two crucial, and usually mutually exclusive, properties of the operator $D$ : the absence of additive

\footnotetext{
${ }^{1}$ E-mail address: twchiu@ @ phys.ntu.edu.tw

${ }^{2}$ Permanent address: Institute for Nuclear Research of the Russian Academy of Sciences, 117312 Moscow, Russia. E-mail address: zenkin@al20.inr.troitsk.ru
}

mass renormalization and reproducing correct anomaly, in particular the Atiyah-Singer index theorem. However, as shown in Ref. [7], only the GW relation itself is not sufficient to guarantee that any $D$ satisfying it reproduces the index theorem on the lattice. A necessary condition for the solutions $D$ of Eq. (1) to have nonzero index in topologically nontrivial sectors has been formulated in Ref. [7]. In topologically trivial sector the general solution of Eq. (1) is given in terms of the chirally invariant Dirac operator $D_{c}[7]$ :

$D=\frac{D_{c}}{1+r D_{c}}, \quad D_{c} \gamma_{5}+\gamma_{5} D_{c}=0$.

In this paper we demonstrate that although the form (2) forbids the additive mass corrections in the fermion propagator determined by $D$, it does not ensure the perturbative stability of the pole of the propagator. As we show, the question whether the pole is stable under radiative corrections or not is determined solely by the structure of chirally invari- 
ant operator $D_{c}$. We illustrate this by explicit examples. We also show that in the absence of gauge interactions the Yukawa models formulated by Lüscher [4] with the GW-Dirac operators $D$ are equivalent to the ordinary Yukawa models defined with the corresponding operators $D_{c}$.

In Section 2, we discuss the basic properties of $D_{c}$ and consider three explicit examples for which the operator $D$ for free fermions is local in the continuum limit. In Section 3 we examine the fermion self-energy determined by these operators, and in Section 4 discuss the Yukawa models. Section 5 is a summary.

\section{Explicit solutions of the Ginsparg-Wilson rela- tion}

For $D$ is nonsingular, according to Eq. (2), the problem of constructing explicit solutions of $D$ reduces to finding a proper realization of the chirally invariant Dirac operator $D_{c}$. We are interested in those $D$ which are local at least in the continuum limit, i.e. $D_{m n}$ vanishes at $a \rightarrow 0$ for any finite physical distance $a|m-n|$. This implies that at large $|m-n|, D_{m n}$ should fall off faster than $|m-n|^{-1}$. On the regular lattice this means that for free fermions the Fourier transform of $D_{m n}, D(p)$, should be a continuous periodic function of momentum $p$, and the smoother the function, the faster $D_{m n}$ falls off with $|m-n|$. This imposes certain limitations to the form of $D_{c}: D_{c}(p)$ should be either continuous, or may have some singularities, but should not have finite discontinuities.

According to the Nielsen-Ninomiya theorem [8] any continuous $D_{c}(p)$ suffers from species doubling, so $D(p)$ constructed via Eq. (2) with such $D_{c}$ will suffer from the doubling too ${ }^{3}$. To avoid species doubling in $D_{c}$, and therefore in $D, D_{c}(p)$ should be discontinuous, i.e. $D_{c}$ should be nonlocal in position space. Such operators can be classified according to the behaviour of $D_{c}(p)$ at the boundary of the

\footnotetext{
${ }^{3}$ The simplest example of such $D$ is that constructed with the naive Dirac operator $D_{c}(p)=i \gamma_{\mu} \bar{p}_{\mu}$. This example clearly demonstrates that the GW relation is not a remedy for the species doubling.
}

Brillouin zone, where $D_{c}(p)$ has either finite or infinite discontinuities. In order to have $D(p)$ continuous, one should reject the $D_{c}(p)$ with finite discontinuities, like the operators involving SLAC derivative [9]. At present we know only three examples of explicit $D_{c}$ whose Fourier transform in the free fermion limit has infinite discontinuities.

(I) The first example is the action proposed by Rebbi in Ref. [10]:

$D_{c}(p)=i \gamma_{\mu} \bar{p}_{\mu} \frac{\hat{p}^{2}}{\bar{p}^{2}}, \quad \bar{p}_{\mu}=\sin p_{\mu}, \quad \hat{p}_{\mu}=2 \sin \frac{p_{\mu}}{2}$.

$D_{c}(p)$ is singular at those points at the boundary of the Brillouine zone where the naive Dirac operator $i \gamma_{\mu} \bar{p}_{\mu}$ has zeroes which produce species doubling. In the presence of gauge field, $D_{c}$ takes the form

$$
\begin{aligned}
D_{c}= & 4\left\{\gamma_{\mu}\left(\nabla_{\mu}^{+}+\nabla_{\mu}^{-}\right),\right. \\
& \left.\left(-\nabla_{\nu}^{-} \nabla_{\nu}^{+}+c g \sigma_{\nu \sigma} F_{\nu \sigma}\right)^{-1}\right\}^{-1},
\end{aligned}
$$

where

$\nabla_{\mu m n}^{+}=U_{m, m+\hat{\mu}} \delta_{m+\hat{\mu}, n}-\delta_{m, n}$,

$\nabla_{\mu m n}^{-}=\delta_{m, n}-U_{m, m-\hat{\mu}} \delta_{m-\hat{\mu}, n}$,

$U_{m, m \pm \hat{\mu}}=\exp \left[ \pm i g A_{\mu}(n \pm \hat{\mu} / 2)\right], \quad \sigma_{\mu \nu}=$ $i\left[\gamma_{\mu}, \gamma_{\nu}\right] / 2, F_{\mu \nu}$ is a lattice transcription of the field tensor, $c$ is a constant which in Ref. [10] has been chosen equal to $1 / 2$, and $\{A, B\}=A B+B A$.

(II) The second example is the Dirac operator which follows from the construction of the fermion path integral for the Weyl ordering [11]:

$D_{c}(p)=i \gamma_{\mu} \tilde{p}_{\mu}, \quad \tilde{p}_{\mu}=2 \tan \frac{p_{\mu}}{2}$.

In this case $D_{c}(p)$ is singular at the whole Brillouin boundary. In the position space it can be written in the form

$D_{c}=\sum_{\mu} \gamma_{\mu}\left\{\left(\nabla_{\mu}^{+}+\nabla_{\mu}^{-}\right),\left(4+\nabla_{\mu}^{+}-\nabla_{\mu}^{-}\right)^{-1}\right\}$.

(III) Our third example is the chirally invariant operator $D_{c}$ deduced from the overlap-Dirac operator proposed by Neuberger in Ref. [3]. This $D$ 
satisfies Eq. (1) for $r \in(1 / 4, \infty)$ and therefore has the form of Eq. (2) with

$$
\begin{aligned}
D_{c}(p)= & i \gamma_{\mu} \bar{p}_{\mu} \frac{2 M}{\bar{p}^{2}} \\
& \times\left[\frac{1}{2} \hat{p}^{2}-M+\sqrt{\bar{p}^{2}+\left(\frac{1}{2} \hat{p}^{2}-M\right)^{2}}\right],
\end{aligned}
$$

where $M \in(0,2)$ is intrinsic parameter of the formulation related to $r$ as $M=(2 r)^{-1} . D_{c}$ in (8) has singularities at the same points of the Brillouin boundary as Rebbi's operator (3). In the presence of gauge interactions, $D_{c}$ can be written as

$D_{c}=2 M \frac{1+V}{1-V}, \quad V=D_{W} \frac{1}{\sqrt{D_{W}^{\dagger} D_{W}}}$,

where $D_{W}$ is the Wilson operator with negative mass $-M$,

$D_{W}=\frac{1}{2}\left[\gamma_{\mu}\left(\nabla_{\mu}^{+}+\nabla_{\mu}^{-}\right)-\nabla_{\nu}^{-} \nabla_{\nu}^{+}\right]-M$.

In terms of the unitary operator $V$, Eq. (2) takes the form

$$
D=M(1+V) \text {. }
$$

\section{Fermionic self energy}

\subsection{General structure}

When $D$ is nonsingular, Eq. (2) is equivalent to $D^{-1}=D_{c}^{-1}+r$.

Then the fermion propagator in the presence of dynamical gauge fields is

$\left\langle D^{-1}\right\rangle=\left\langle D_{c}^{-1}\right\rangle+r$,

where the brackets denote normalized averaging over all topologically trivial gauge configurations. In order to simplify the analysis of self-energy operators, we limit ourselves to the quenched approximation, i.e. $\operatorname{det} D=1$ in the measure of averaging in (13). Then in the momentum space the full propagators $\left\langle D^{-1}\right\rangle$ and $\left\langle D_{c}^{-1}\right\rangle$ can be written as

$$
\begin{aligned}
& \left\langle D^{-1}\right\rangle(p)=\frac{1}{D(p)+\Sigma_{G W}(p)}, \\
& \left\langle D_{c}^{-1}\right\rangle(p)=\frac{1}{D_{c}(p)+\Sigma_{c}(p)},
\end{aligned}
$$

where $\Sigma_{G W}(p)$ and $\Sigma_{c}(p)$ are the fermion self-energy operators related to the Dirac operators $D$ and $D_{c}$, respectively. Since $D(p)$ and $D_{c}(p)$ vanish at $p=0$, it follows from (13) and (14) that at $p \rightarrow 0$

$\Sigma_{G W}(p) \rightarrow \frac{\Sigma_{c}(p)}{1+r \Sigma_{c}(p)}$.

Operator $D_{c}$ is chirally invariant, so is $\Sigma_{c}$. This implies that it contains only odd combinations of $\gamma$-matrices. In the simplest case one has

$\Sigma_{c}=i \gamma^{\mu} \Sigma_{c}^{\mu}$,

$\Sigma_{G W}(0)=\frac{i \gamma^{\mu} \Sigma_{c}^{\mu}(0)}{1+r^{2} \Sigma_{c}^{2}(0)}+\frac{r \Sigma_{c}^{2}(0)}{1+r^{2} \Sigma_{c}^{2}(0)}$.

If $\Sigma_{c}(0)=0$, the poles in the propagators are not shifted, and radiative corrections lead only to a renormalization of the fermion wavefunctions. However, because of the nonlocality of $D_{c}, \Sigma_{c}(0)$ may not be always zero. Then the poles of both propagators are shifted, and in $\left\langle D^{-1}\right\rangle$ a scalar additive term

$\delta m=\frac{r \Sigma_{c}^{2}(0)}{1+r^{2} \Sigma_{c}^{2}(0)}$

is generated.

In the perturbation theory $\Sigma=\sum_{n} g^{2 n} \Sigma^{(2 n)}$, and in the lowest order one has

$\Sigma_{G W}^{(2)}(p \rightarrow 0) \rightarrow \Sigma_{c}^{(2)}(p \rightarrow 0)$.

So there is no $\delta m$ generated in the lowest order in the perturbation theory, however the pole still may be shifted.

\section{2. $\Sigma(0)$ : one loop analysis}

In the perturbation theory one has

$D=D^{(0)}+g D^{(1)}+g^{2} D^{(2)}+O\left(g^{4}\right)$,

where in the momentum space $D^{(1,2)}$ can be written in the form

$$
\begin{aligned}
D^{(1)}\left(p^{\prime}, p\right)= & \int_{q} \delta_{2 \pi}\left(p^{\prime}-p-q\right) V_{\mu}(p, q) A_{\mu}(q), \\
D^{(2)}\left(p^{\prime}, p\right)= & \int_{q^{\prime}, q} \delta_{2 \pi}\left(p^{\prime}-p-q^{\prime}-q\right) \\
& \times V_{\mu \nu}\left(p, q^{\prime}, q\right) A_{\mu}\left(q^{\prime}\right) A_{\nu}(q),
\end{aligned}
$$


with $\int_{q}=\int_{(-\pi / a, \pi / a]^{4}} d^{4} q /(2 \pi)^{4}$ and $\delta_{2 \pi}(q)$ is $2 \pi$-periodic $\delta$-function. Then

$$
\begin{aligned}
& \Sigma^{(2)}(p) \\
& \quad=C_{2} \int_{q}\left[-V_{\mu}(p+q,-q) \frac{1}{D^{(0)}(p+q)} V_{\nu}(p, q)\right. \\
& \left.\quad+V_{\mu \nu}(p,-q, q)\right] \Delta_{\mu \nu}(q) \\
& =\Sigma_{1}(p)+\Sigma_{2}(p),
\end{aligned}
$$

where $C_{2}$ is the Casimir operator, and $\Delta_{\mu \nu}$ comes from the gauge field propagator; in the covariant gauge it has the form

$\Delta_{\mu \nu}(q)=\delta_{\mu \nu} \frac{1}{\hat{q}^{2}}-(1-\alpha) \hat{q}_{\mu} \hat{q}_{\nu} \frac{1}{\left(\hat{q}^{2}\right)^{2}}$.

In (21) the first term in the integrand is the lattice counterpart of the continuum self-energy diagram, while the second term corresponds to the tadpole diagram, and they are denoted by $\Sigma_{1}$ and $\Sigma_{2}$, respectively.

From the Ward identities for the vertices

$$
\begin{aligned}
& V_{\mu}(p, q) \hat{q}_{\mu}=D^{(0)}(p+q)-D^{(0)}(p), \\
& V_{\mu \nu}\left(p, q^{\prime}, q\right) \hat{q}_{\mu}^{\prime} \hat{q}_{\nu} \\
& \quad=\frac{1}{2}\left[D^{(0)}\left(p+q^{\prime}+q\right)-D^{(0)}\left(p+q^{\prime}\right)\right. \\
& \left.\quad-D^{(0)}(p+q)+D^{(0)}(p)\right],
\end{aligned}
$$

it follows that $\Sigma^{(2)}(p \rightarrow 0)$ does not depend on gauge fixing parameter $\alpha$ in (22), so we fix the Feynman gauge $\alpha=1$.

The explicit form of the vertices for each of our examples are derived from the corresponding expressions for the operator $D$ through the expansion (19). By virtue of relation (18) one can instead of $D$ use only its chirally invariant part $D_{c}$. Except for the operator in Eq. (7), the explicit expressions for the vertices are quite cumbersome, so we present here only the results for $\Sigma^{(2)}(p \rightarrow 0)$.

(I) $D_{c}$ in Eq. (4): In this case we can use the results obtained in Ref. [12]. Then we get

$$
\Sigma_{c}^{(2)}(p \rightarrow 0) \rightarrow i \gamma_{\mu} p_{\mu} \frac{1}{p^{2}}\left(A-c^{2} B\right),
$$

where $A$ and $B$ are certain finite momentum integrals. $B$ is determined by the $c$-part in Eq. (4) and depends on the lattice transcription of the field tensor $F_{\mu \nu}$. It is evident that we cannot obtain $\Sigma_{c}^{(2)}(p \rightarrow 0)=0$ without fine-tuning the parameter $c$. Thus, this example demonstrates perturbative instability of the pole of the fermion propagator.

(II) $D_{c}$ in Eq. (7): $\Sigma_{c}^{(2)}(0)=0$, both $\Sigma_{c 1}(0)$ and $\Sigma_{c 2}(0)$ vanish.

(III) $D$ in Eq. (11): $\Sigma_{G W}^{(2)}(0)=0{ }^{4}$. In this case it is the result of cancellation of nonzero $\Sigma_{G W 1}(0)$ and $\Sigma_{G W 2}(0)$ :

$$
\begin{aligned}
\Sigma_{G W 1}(0)= & -\Sigma_{G W 2}(0) \\
= & -C_{2} \int_{q} \frac{M}{\left(M+\sqrt{\bar{p}^{2}+B^{2}(p)}\right)^{2}} \\
& \times \sum_{\mu}\left\{V_{\mu}^{2}(0, q)[1-\bar{B}(q)]\right. \\
& +W_{\mu}^{2}(0, q)[1+\bar{B}(q)] \\
& \left.+2 V_{\mu}(0, q) W_{\mu}(0, q) \bar{C}_{\mu}(q)\right\} \frac{1}{\hat{q}^{2}},
\end{aligned}
$$

where

$$
\begin{aligned}
& B(q)=\frac{1}{2} \hat{p}^{2}-M, \quad \bar{B}(q)=\frac{B(q)}{\sqrt{\bar{p}^{2}+B^{2}(p)}}, \\
& \bar{C}_{\mu}(q)=\frac{\bar{p}_{\mu}}{\sqrt{\bar{p}^{2}+B^{2}(p)}}, \\
& V_{\mu}(p, q)=\cos \left(p_{\mu}+\frac{1}{2} q_{\mu}\right), \\
& W_{\mu}(p, q)=\sin \left(p_{\mu}+\frac{1}{2} q_{\mu}\right) .
\end{aligned}
$$

\section{3. $\Sigma(0)$ : mean field estimate}

Let us estimate now $\Sigma(0)$ in a mean-field approximation in which $\left\langle U_{m, m \pm \hat{\mu}}\right\rangle=\langle U\rangle=$ const. $\langle 1$ and $\langle F(U)\rangle=F(\langle U\rangle)$ for any matrix $F(U)$. This approximation corresponds to the summation of certain subsets of the tadpole diagrams in the Feynman gauge. We have no illusions about its accuracy,

\footnotetext{
${ }^{4}$ This result is anticipated in view of previous results obtained in the domain wall and the overlap formulations [13].
} 
however we find it quite instructive. For our examples we have:

(I) $\Sigma_{c}(p \rightarrow 0)$ has the same form as the r.h.s. of Eq.

(24) with $A=2(1 /\langle U\rangle-1)$, and $B=0$. From

Eq. (17) it follows that $\delta m=1 / r$ for any $\langle U\rangle<1$.

(II) $\Sigma_{c}(0)=0, \delta m=0$ for any $\langle U\rangle<1$.

(III) $\Sigma_{G W}(0)=0, \delta m=0$, if $\langle U\rangle>1-M / 4$. Note that this result agrees with that obtained in the domain wall fermions in Ref. [14], as well as with the reasonings of Ref. [15].

\section{Yukawa models}

General solutions of the GW relation in terms of the chirally invariant operator $D_{c}$ given in (2) suggests a simple interpretation of the chirally and flavour invariant construction of Yukawa models proposed in Ref. [4]. In this secton we turn off the gauge interactions.

The fermion action in this formulation is defined as

$S=\sum_{n}\left[\bar{\psi} D \psi-r^{-1} \bar{\chi} \chi+y(\bar{\psi}+\bar{\chi}) \Phi(\psi+\chi)\right]$,

where $\Phi=\frac{1}{2}\left(1+\gamma_{5}\right) \phi^{\dagger}+\frac{1}{2}\left(1-\gamma_{5}\right) \phi$ is the Higgs field, $y$ is the Yukawa coupling, and $\bar{\chi}, \chi$ are auxiliary non-propagating fermion fields. This action is invariant under the following infinitesimal variations

$$
\begin{aligned}
& \delta \psi=\alpha \gamma_{5}[(1-r D) \psi+\chi], \quad \delta \chi=\alpha \gamma_{5} r D \psi, \\
& \delta \bar{\psi}=[\bar{\psi}(1-r D)+\bar{\chi}] \alpha \gamma_{5}, \quad \delta \bar{\chi}=\bar{\psi} r D \alpha \gamma_{5}, \\
& \delta \Phi=-\left\{\alpha \gamma_{5}, \Phi\right\} .
\end{aligned}
$$

where $\alpha$ is an infinitesimal global parameter which may be nontrivial in the flavour space. Making obvious change of variables and taking into account Eq. (2) we obtain

$$
\begin{gathered}
Z[\Phi]=\int \prod_{n} d \psi d \bar{\psi} d \chi d \bar{\chi} \exp (-S) \\
=C \int \prod_{n} d \psi^{\prime} d \bar{\psi}^{\prime} \exp \left(-S^{\prime}\right), \\
S^{\prime}=\sum_{n} \bar{\psi}^{\prime}\left(D_{c}+y \Phi\right) \psi^{\prime},
\end{gathered}
$$

where $C$ is irrelevant constant, and the action $S^{\prime}$ is chirally invariant in the usual sense, i.e. invariant under the variations

$$
\delta \psi^{\prime}=\alpha \gamma_{5} \psi^{\prime}, \quad \delta \bar{\psi}^{\prime}=\bar{\psi}^{\prime} \alpha \gamma_{5}, \quad \delta \Phi=-\left\{\alpha \gamma_{5}, \Phi\right\} .
$$

Thus, the Yukawa model (27) with GW-Dirac operator $D$ is equivalent to the usual Yukawa model with the chirally invariant Dirac operator $D_{c}$.

System (29) can be easily examined within the mean-field approximation developed in Ref. [16]. In particular, in the case of $\phi^{\dagger} \phi=1$ and scalar hopping parameter $\kappa \geq 0$ its critical lines in this approximation are determined by only two constants $G^{W}=$ $\int_{p}\left[D_{c}^{\dagger}(p) D_{c}(p)\right]^{-1}$ and $G^{S}=\int_{p} D_{c}^{\dagger}(p) D_{c}(p)$ (for more detail see Ref. [16]).

\section{Summary}

We have demonstrated that in topologically trivial sector some important properties of a Dirac operator $D$ satisfying the $\mathrm{GW}$ relation (1) are determined by the non-local chirally invariant Dirac operator $D_{c}$ introduced in (2). In particular, in the absence of the gauge interactions the Yukawa model constructed by Lüscher [4] is shown to be equivalent to the ordinary Yukawa model defined with the operator $D_{c}$. In addition to the overlap-Dirac operator [3], we constructed two new explicit solutions of the GW relation and examined the fermionic self-energy operators of these three operators respectively. We demonstrate that a lattice Dirac operator $D$ satisfying the GW relation does not necessarily provide the perturbative stability of the pole of the fermion propagator, and this property solely depends on the structure of the corresponding chirally invariant Dirac operator $D_{c}$. Among the three examples we have considered only two [Eqs. (7) and (9)] maintain perturbative stability of the pole of the fermion propagator, while the operator Eq. (4) fails without fine tuning the parameter $c$, see Eq. (24).

On the other hand, in the topologically non-trivial sectors, the necessary condition for a Dirac operator $D$ satisfying $\mathrm{GW}$ relation to have non-zero index is $\operatorname{det}(1-r D)=0$, that is equivalent to the non-existence of the chirally invariant operator $D_{c}$ in topo- 
logically nontrivial gauge field background [7]. This condition is satisfied only by the $D_{c}$ of the overlapDirac operator (9)-(11) (for more detail see Refs. $[5,7]$,$) . We also note that in two-dimensional gauge$ models operators $D_{c}$ in (4) and (7) have no genuine zeromodes in topologically non-trivial sectors, similar to that of the naive Dirac operator [7].

\section{Acknowledgements}

This work is supported by the National Science Council, R.O.C. under the grant number NSC872112-M002-013.

\section{References}

[1] P.H. Ginsparg, K.G. Wilson, Phys. Rev. D 25 (1982) 2649.

[2] P. Hasenfratz, Nucl. Phys. B (Proc. Suppl.) 63A-C (1998) 53; P. Hasenfratz, V. Laliena, F. Niedermayer, The Index Theorem in QCD with a finite cutoff, hep-lat/9801021; P. Hasenfratz, Lattice QCD without tuning, mixing and current renormalization, hep-lat/9802007.
[3] H. Neuberger, Phys. Lett. B 417 (1998) 141; More about exactly massless quarks on the lattice, hep-lat/9801031.

[4] M. Lüscher, Exact chiral symmetry on the lattice and the Ginsparg-Wilson relation, hep-lat/9802011.

[5] T.W. Chiu, Topological Charge and the Spectrum of Exactly Massless Fermion on the Lattice, hep-lat/9804016, to be published in Phys. Rev. D.

[6] S. Chandrasekharan, Lattice QCD with Ginsparg-Wilson fermions, hep-lat/9805015.

[7] T.W. Chiu, S.V. Zenkin, On solutions of the Ginsparg-Wilson relation, hep-lat/9806019.

[8] H.B. Nielsen, M. Ninomiya, Nucl. Phys. B 185 (1981) 20 [E: B 195 (1982) 541]; B 193 (1981) 173.

[9] S.D. Drell, M. Weinstein, S. Yankielowicz, Phys. Rev. D 14 (1996) 1627.

[10] C. Rebbi, Phys. Lett. B 186 (1987) 200.

[11] S.V. Zenkin, Sov. Phys. Lebedev Inst. Rep. 9 (1988) 10; Mod. Phys. Lett. A 6 (1991) 151.

[12] G.T. Bodwin, E.V. Kovacs, Phys. Lett. B 193 (1987) 283.

[13] S. Aoki, Y. Taniguchi, One-loop calculation in lattice QCD with domain wall quarks, hep-lat/9711004; H. Neuberger, Y. Kikukawa, A. Yamada, Exponential suppression of radiatively induced mass in the trancated overlap, heplat/9712022.

[14] S. Aoki, K. Nagai, S.V. Zenkin, Nucl. Phys. B (Proc. Suppl.) 63A-C (1998) 602.

[15] H. Neuberger, Phys. Rev. D 57 (1998) 5417.

[16] S. Tominaga, S.V. Zenkin, Phys. Rev. D 50 (1994) 2604; S.V. Zenkin, Phys. Rev. D 53 (1996) 6674. 